Produto \& Produção, vol. 13 n. 1, p. 93-113 fev. 2012

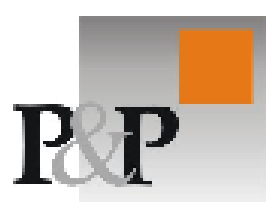

\title{
Fatores críticos da agilidade no gerenciamento de projetos de desenvolvimento de novos produtos
}

Recebido em 13/12/2011. Aceito em 20/01/2012.

Luís Fernando Magnanini Almeida

Universidade Federal de São Carlos - UFSCar

Depto. de Engenharia de Produção

luisfernando@dep.ufscar.br

\section{Edivandro Carlos Conforto}

Escola de Engenharia de São Carlos - Universidade de São Paulo

Grupo de Engenharia Integrada

econfort@sc.usp.br

\section{Sérgio Luís Silva}

Universidade Federal de São Carlos - UFSCar

Depto. Ciências Informação/Programa de Pós-Graduação em Engenharia de Produção

sergiol@ufscar.br

\section{Daniel Capaldo Amaral}

Escola de Engenharia de São Carlos - Universidade de São Paulo amaral@sc.usp.br

As limitações da abordagem tradicional de Gerenciamento de Projetos (GP) quando utilizada no Desenvolvimento de Produtos (DP) inovadores contribuíram para a disseminação da teoria de "Gerenciamento Ágil de Projetos" (GAP). Além disso, existe uma lacuna quanto à existência de literatura específica que explora a influencia de alguns fatores no desempenho e uso de práticas e técnicas do APM. Por meio de uma revisão bibliográfica sistemática, este artigo reúne 36 Fatores Críticos da Agilidade (FCA) que podem influenciar no desempenho e uso da abordagem do gerenciamento ágil de projetos. Esses fatores foram selecionados tendo como base textos de diversas áreas como: manufatura, organização, cadeia de suprimentos, gerenciamento de projetos (tradicional e ágil), software e desenvolvimento de produtos. Esta lista de FCAs e a análise dos resultados é um primeiro esforço para sistematizar um conjunto de fatores que podem influenciar a adoção e desempenho do APM. Também pode ser útil para estudos empíricos comparativos entre as duas teorias APM e Tradicional.

Palavras chave: Fatores Críticos da Agilidade, Gerenciamento Ágil de Projetos, Gerenciamento de Projetos 
The restrictions of "traditional" project management when applied to innovative product development have been contributed to the "Agile Project Management" (APM) theory dissemination. Few studies have focused on the exploration of APM benefits when applied to new product development projects. Additionally, there is a lack of specific literature considering the influence of some factors in the use and performance of agile project management practices and techniques. By applying systematic literature review this article presents a set of 36 Agility Critical Factor's (ACF) that can influence the performance of agile project management practices and techniques. The factors were selected based on a set of journal articles published in manufacturing, organization, supply chain, project management and product development areas. The final list and results analysis' are the first effort to systematize a set of ACFs for APM, and also can be useful for empirical studies that pursue comparative analyses between APM and "traditional" theory.

Keywords: Agility Critical Factor's, Agile Project Management, Project Management

\section{INTRODUÇÃO}

Desde a década de 1950 a teoria de gerenciamento de projetos tem evoluído consistentemente. Evidência dessa evolução são o surgimento e a consolidação das associações que atuam na padronização e disseminação das práticas de GP e dos "corpos de conhecimento" ou "Body of Knowledge (BOKs)" (KIOPPENBORG; OPFER, 2002; KOLLTVEIT; KARLSEN; GRONHAUG, 2007; SHENHAR; DVIR, 2007 apud EDER et al, 2010).

Essa evolução é natural. Sauser et al (2009) explica que os projetos se tornaram uma atividade central para a maioria das organizações devido à crescente necessidade de desenvolver novos produtos e serviços. Segundo esses autores, os projetos podem ter dois tipos de falhas, as técnicas e as gerenciais. Grande parte das falhas gerenciais está relacionada às escolhas da abordagem de GP, e, por conseguinte, das práticas gerenciais, que podem ser inadequadas para aquele contexto. Essa opinião é compartilhada por Shenhar (2001) que afirma que poucos autores tentaram classificar os projetos de acordo com um tipo específico e destes, raros foram os casos que tentaram investigar a correlação das práticas de gestão adotadas e o tipo de projeto. Em sua maioria, os projetos são gerenciados da mesma forma, sem a devida diferenciação dos projetos, da compreensão dos fatores críticas e das diferenças entre as práticas de gestão, motivo pelo qual o autor atribui grande quantidade de insucessos.

Logo, pela falta de diferenciação entre os projetos e do uso de práticas adequadas para cada tipo, geralmente são adotadas as denominadas "melhores práticas" de gerenciamento, sendo elas reunidas a partir da análise de um grande número de projetos em diferentes áreas. O problema não está nas práticas em si, mas sim, no seu uso generalista, principalmente no desenvolvimento de produtos inovadores. Este o uso de práticas e técnicas de GP de forma genérica, sem a devida adaptação, tem gerado críticas explicitadas por diversos autores (DAWSON; DAWSON, 1998; PERMINOVA; GUSTAFSSON; WIKSTRÖM, 2008; WILLIAMS, 1999). Essas inquietações são ainda mais constantes nos gestores de 
desenvolvimento de software. Nessa área está o marco inicial da abordagem de gerenciamento ágil de projetos, representado na forma de um documento, intitulado "Manifesto para Desenvolvimento Ágil de Software (do inglês "Manifesto for Agile Software Development") que foi elaborado por um grupo de profissionais e acadêmicos em 2001 (BECK et al, 2001).

Em relação aos produtos manufaturados, Ebert e Man (2008) defendem a necessidade de melhoria no gerenciamento de projetos, desenvolvimento de novos produtos e processos de engenharia como ações cruciais para empresas permanecerem competitivas em ambientes de mudança. Assim, a partir da criação e disseminação da abordagem do GAP, tem-se uma alternativa para gerenciar projetos inovadores com foco na simplicidade, valorização das pessoas, interações com o cliente, e utilização de técnicas e ferramentas visuais de gestão (AUGUSTINE, 2005; CHIN, 2004; COHN, 2005; HIGHSMITH, 2004; AMARAL et al, 2011).

Em comum, os textos sobre GAP apresentam práticas que podem trazer benefícios frente às formas "tradicionais" de GP. As práticas e técnicas, segundo o esses autores, seriam mais apropriadas para os ambientes dinâmicos de negócios. Contudo, existem lacunas nessa teoria. Um exemplo disso é a própria definição do termo "gerenciamento ágil de projetos" para o contexto de desenvolvimento de produtos. Nesse sentido, pesquisas têm contribuído para sistematizar e aprofundar o conhecimento nessa área. A definição adotada nesse texto segue a proposta de Amaral et al (2011): "O gerenciamento ágil de projetos é uma abordagem fundamentada em um conjunto de princípios, cujo objetivo é tornar o processo de gerenciamento de projetos mais simples, flexível e iterativo, de forma a obter melhores resultados em desempenho (tempo, custo e qualidade), menos esforço em gerenciamento e maiores níveis de inovação e agregação de valor para o cliente".

Essa abordagem é mais indicada para ambientes turbulentos de negócio, ou seja, aqueles nos quais as empresas enfrentam dificuldade em se prever o futuro e precisam gerenciar grandes incertezas e desafios no planejamento e controle dos projetos. Nesse sentido, as técnicas ditas "tradicionais" de GP têm apresentado limitações (SUIKKI; TROMSTEDT; HAAPASALO, 2006). Estudos recentes buscam explorar o potencial do GAP para o desenvolvimento de produtos inovadores, como é o caso de Conforto e Amaral (2009, 2010) e Almeida et al (2010). Em comum, esses estudos apontam para a influencia de um conjunto de fatores, muitos dos quais não tem relação direta com as práticas e técnicas de gerenciamento de projetos, mas sim, como a forma com que são utilizadas, e o ambiente de projeto. Estudos na área de software também evidenciaram o efeito de alguns fatores críticos que impactaram o gerenciamento de projetos inovadores (MAFAKHERI; NASIRI; MOUSAVI, 2008; QUMER; SELLERS, 2008).

Esses fatores, denominados nesse estudo de "Fatores Críticos da Agilidade", podem limitar ou potencializar o desempenho de um conjunto de práticas de GP e, assim. impactar diretamente no resultado do projeto. Existe, portanto, uma lacuna na teoria quanto à identificação e teste empírico da influencia desses fatores no gerenciamento ágil de projetos. A identificação, categorização e definição desses fatores é o primeiro passo para melhor compreender qual sua influencia no desempenho das práticas, técnicas e ferramentas propostas segundo a abordagem do GAP.

A partir de uma revisão bibliográfica sistemática este artigo apresenta um conjunto de fatores críticos da agilidade, agrupados em quatro categorias: organização, processo, time de projeto e produto. A principal contribuição está na 
identificação e análise desses fatores, utilizando como critério a quantidade de citações na teoria. Busca-se contribuir com o embasamento teórico necessário para futuros estudos empíricos dos efeitos desses fatores na adoção de práticas de gerenciamento ágil de projetos.

\section{FATORES CRÍTICOS DA AGILIDADE NO GAP}

Conforme já mencionado o marco inicial da teoria GAP foi o manifesto para desenvolvimento ágil de software (BECK et al, 2001). Nessa área vários métodos ágeis foram criados, como: Extreme Programming (XP), Scrum, Dynamic System Development Method (DSDM), Adaptative Software Programming (ASP), Crystal, Feature-Driven Development (FDD), Pragmatic Programming (EDER et al, 2010; $\mathrm{CHOW}$; CAO, 2008). Além desses métodos, com foco exclusivo na área de software, outras propostas tem ganhado espaço, pois buscam atender empresas que desenvolvem produtos manufaturados, mas que também sofrem influência de incertezas, mudanças constantes e buscam a inovação como estratégia competitiva. Uma dessas propostas é o método IVPM2 (Iterative and Visual Project Management Method) proposto por Conforto e Amaral (2009; 2010) descrito em Amaral et al (2011).

A pesquisa realizada por Conforto e Amaral (2010) em duas empresas de base tecnológica teve por objetivo desenvolver e implantar o IVPM2. Em ambas as empresas, os participantes do estudo (gerentes de projeto e membros de equipe) identificaram alguns dos principais fatores que influenciaram na implantação e uso das práticas, técnicas e ferramentas indicadas nesse método, com destaque para: cultura organizacional voltada pra a utilização da abordagem GAP; Formação da equipe e sua resistência na adoção de técnicas de gerenciamento de projetos; Falta de conhecimento sobre os conceitos de gerenciamento ágil de projetos.

O estudo de Conforto e Amaral (2010), portanto, aponta para a necessidade de melhor explorar os fatores internos e externos que podem impactar no uso da abordagem de gerenciamento ágil de projetos. Além disso, outro aspecto que merece ser investigado é o próprio conceito de agilidade, disseminado na teoria GAP, mas que foi cunhado na área de manufatura (YUSUF; SARHADI; GUNASEKARAN, 1999) e rapidamente foi adotado em outras áreas como organizações e cadeia de suprimentos (BOTTANI, 2009; BUSTELO et al, 2007; DOVE, 1999; GANGULY et al, 2009; YUSUF et al, 1999; ZHANG; SHARIF, 1999).

No início da década de 2000 a área de gerenciamento de projetos também passou a utilizar o termo "agilidade" (BECK et al, 2001). Contudo, o seu emprego de forma genérica em textos de GAP voltados para a área de software tem sido alvo de pesquisas e estudos para melhor compreender sua relação com os métodos de gerenciamento ágil de projetos (ALMEIDA et al, 2010; MAFAKHERI; NASIRI; MOUSAVI, 2008; QUMER; SELLERS, 2008). Essa falta de precisão na definição do termo fica evidente quando se observa e analisa as definições encontradas na literatura.

Nesse sentido um primeiro esforço empreendido para melhor compreensão do termo é descrito em Eder et al (2010) que define "agilidade" como: "[...] a habilidade de se adquirir velocidade e flexibilidade no gerenciamento de projetos por meio da adoção de práticas de gestão adequadas ao ambiente e tipo de projeto". Apesar das limitações e da necessidade de estudos mais aprofundados sobre o 
tema, esta definição será adotada neste trabalho, como sinônimo para desempenho segundo a aplicação do gerenciamento ágil de projetos.

A partir dessa definição do termo "agilidade", assume-se que o desempenho esperado pelo uso de práticas advindas da teoria do GAP, por exemplo, ganhos em agilidade e flexibilidade, pode ser diretamente influenciado por fatores internos ou externos à organização. Ou seja, existem vários FCA, conforme proposto neste artigo, que podem influenciar no uso das práticas de GAP e, por conseguinte, na "agilidade".

Antes de apresentar a definição de FCA é importante fazer uma distinção entre os estudos relacionados aos fatores críticos de sucesso (FCS) em projetos, que tratam do sucesso do projeto como um todo por meio da identificação e gerenciamento dos fatores críticos. Segundo Chow e Cao (2008) o conceito de FCS é uma abordagem para identificar e medir o desempenho de uma organização, tendo origem nos trabalhos desenvolvidos por Rockart $(1979 ; 1981)$ que define os FCS como sendo: "um número limitado de áreas com resultados satisfatórios que asseguram um desempenho competitivo de sucesso para o indivíduo, departamento ou organização". Os FCS seriam as áreas chaves nas quais as coisas "devem dar certo" para que o negócio prospere a as metas sejam cumpridas.

Contudo, esse artigo tem foco na "agilidade" do processo de GP e não no sucesso do projeto como um todo. Sendo assim, o objetivo é apresentar uma lista de fatores que podem impactar no uso de práticas, técnicas e ferramentas provenientes da teoria GAP. Para isso, o conceito de FCS é adaptado redirecionando o foco do sucesso do projeto para a "agilidade" do processo de GP no desenvolvimento de produtos, dando origem ao termo "fatores críticos de agilidade" que é definido como: "fatores internos ou externos à organização que estão relacionados direta ou indiretamente com a agilidade do processo de gerenciamento de projetos no desenvolvimento de produtos podendo impactar positiva ou negativamente no desempenho e uso de determinada prática, técnica ou ferramenta".

Desse modo, é imprescindível para qualquer medida futura ou comparação do desempenho no processo de gerenciamento de projetos identificar e compreender os FCAs, assim como observar sua correlação com as práticas, técnicas e ferramentas de gestão advindas da abordagem do GAP.

\section{MÉTODO}

O estudo foi desenvolvido a partir de uma revisão bibliográfica sistemática (CONFORTO; AMARAL; SILVA, 2011) e foi dividido em quatro etapas:

Etapa 1: Foi realizado um levantamento exploratório com objetivo de identificar lacunas na teoria de GAP, identificar palavras-chave, autores principais e periódicos relevantes para o estudo.

Etapa 2: A partir desse primeiro levantamento, foi definida a string de busca (frase contendo as palavras-chave e operadores lógicos para inserir nas bases de dados e realizar buscas por artigos). A string de busca foi adaptada para cada base de dados diferente, com o objetivo de realizar as buscas de forma individual nos periódicos selecionados. Ao todo foram considerados 87 periódicos.

Etapa 3: Os artigos encontrados passaram por 3 filtros. O Filtro 1 contemplou a leitura do título, resumo e palavras-chave. O critério para inclusão do artigo foi apresentar algum fator crítico para o gerenciamento de projetos de novos produtos. 
No Filtro 2, os artigos selecionados no Filtro 1 foram submetidos à leitura da introdução e conclusão. No Filtro 3, foi realizada a leitura completa do artigo. Os artigos do Filtro 3 também foram submetidos à busca cruzada. Caso tivessem feito referência a outros artigos que pudessem ser importantes para o escopo da pesquisa e que não foram encontrados por meio das strings, esses novos artigos foram selecionados e passaram pelos mesmos filtros, repetindo-se o processo.

Etapa 4: As informações de interesse foram sistematizadas e organizadas. Em seguida conduziu-se a análise dos textos e compilação dos fatores encontrados. Por fim, foram considerados textos que apresentaram fatores críticos segundo o "conceito de agilidade" em áreas como manufatura, cadeia de suprimentos (supply chain), desenvolvimento de produtos, desenvolvimento de software, organizações e gerenciamento de projetos (tradicional e ágil).

Como resultado da revisão sistemática um total de 8.653 artigos tiverem seus títulos lidos e palavras-chave verificados. Em seguida, identificou-se uma amostra de 553 artigos que foram submetidos aos filtros de leitura. Desses, 170 artigos foram considerados aptos a passar para a próxima etapa que consistia em uma leitura completa. Do total de 170 artigos, 112 já foram lidos completamente. A partir da análise das referências desses 112 artigos, por meio de busca cruzada, foram identificados 12 artigos adicionais, totalizando 124 artigos.

Desse montante, 25 artigos apresentaram fatores críticos que tem alguma relação com o desenvolvimento de produtos ou gerenciamento de projetos, sendo esta a amostra de artigos utilizada neste estudo. Os 25 artigos foram agrupados por área de conhecimento, de acordo com o enfoque de cada trabalho. Foram identificadas ao todo 7 áreas, conforme ilustrado na figura 1. O gráfico mostra a distribuição (porcentagem \%) da amostra de artigos segundo as áreas de conhecimento. A área com maior representatividade é Desenvolvimento de Produtos, seguida de Gestão de Organizações (Organizações).



Figura 1 - Distribuição dos artigos selecionados segundo a área do conhecimento

Em seguida os artigos foram agrupados por periódico. A figura 2 ilustra a distribuição dos artigos por periódico. Dos 87 periódicos pesquisados a amostra de 
artigos representa 14 periódicos nos quais foram encontrados artigos relevantes para o presente estudo.

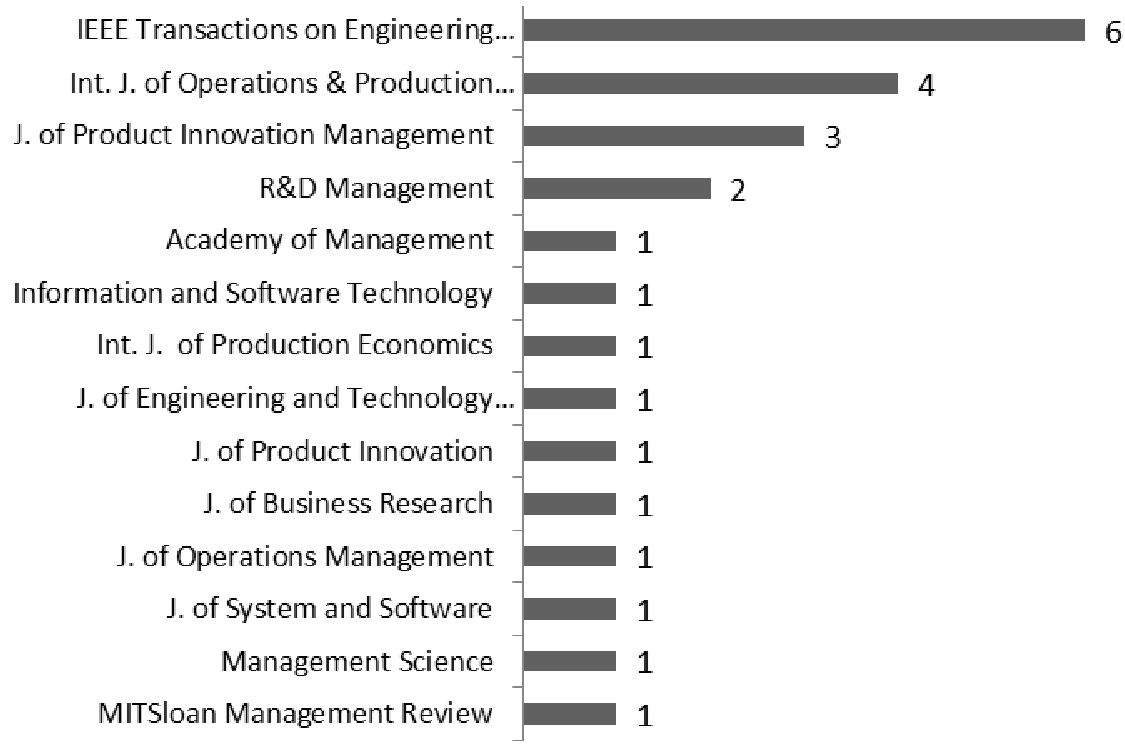

Figura 2 - Distribuição dos artigos selecionados por periódico

A partir dessa base de 25 artigos, foram identificados 36 fatores críticos que podem exercer influência no gerenciamento ágil de projetos de desenvolvimento de novos produtos. Após a identificação e agrupamento desses fatores, foi conduzida uma análise para identificar os fatores críticos mais relevantes, segundo a teoria. Considerou dois critérios: 1) número de áreas em que o fator está presente, ou foi citado (extensão do conceito); 2) quantidade citações dos artigos que apresentaram os fatores críticos. Para o critério 2 foi utilizada a base de dados ISI Web of Knowledge (ISI) e a ferramenta do Google, o Google Scholar (GS). A coleta de dados referente à quantidade de citações foi conduzida no período entre maio e julho de 2011. A Tabela 1 apresenta a classificação dos artigos selecionados neste estudo segundo a quantidade de citações. Esta tabela serviu de base para a aplicação do critério 2, conforme descrito anteriormente.

Tabela 1 - Classificação dos artigos considerados na amostra segundo a quantidade de citações

\begin{tabular}{llccr}
\hline \multicolumn{1}{c}{ Artigos } & $\begin{array}{l}\text { Área do } \\
\text { conhecimento }\end{array}$ & GS & ISI & \multicolumn{1}{c}{$\begin{array}{c}\text { GS + } \\
\text { ISI }\end{array}$} \\
\hline $\begin{array}{l}\text { Kessler e Chakrabarti (1999) apud Chen } \\
\text { et al (2009) }\end{array}$ & $\begin{array}{l}\text { DP } \\
\text { Cadeia de }\end{array}$ & 348 & 155 & 503 \\
$\begin{array}{l}\text { Yusuf, Sarhadi e Gunasekaran (1999) } \\
\text { Tatikonda e Montoya-Weiss (2001) apud }\end{array}$ & $\begin{array}{l}\text { Suprimentos } \\
\text { Chen et al (2009) }\end{array}$ & 263 & 111 & 374 \\
$\begin{array}{l}\text { Sheremata (2000) apud Chen et al } \\
\text { (2009) }\end{array}$ & DP & 180 & 89 & 269 \\
Zirger e Hartley (1996) & DP & 193 & 75 & 268 \\
\hline
\end{tabular}




\begin{tabular}{llrrr}
\hline Lynn et al (2000) apud Chen et al (2009) & DP & 92 & 45 & 137 \\
Verganti (1999) & DP & 89 & 26 & 115 \\
Smith e Reinertsen (1992) & DP & 79 & 22 & 101 \\
Chen et al (2005) & DP & 72 & 23 & 95 \\
Chow e CaO (2008) & Software & 68 & 18 & 86 \\
Matson e McFarlane (1999) & Manufatura & 46 & 16 & 62 \\
Swink (2003) apud Chen et al (2009) & DP & 37 & 13 & 50 \\
Gehani (1995) & Organizações & 32 & 15 & 47 \\
Cornell e Dillon (2001) & GP Tradicional & 13 & 10 & 23 \\
Bustelo, Avella e Fernández (2007) & Manufatura & 13 & 9 & 22 \\
Eppinger e Chitkara (2009) & DP & 0 & 16 & 16 \\
Li e Gima (1999) apud Chen et al (2009) & DP & 14 & 0 & 14 \\
Liu e Yetton (2007) & GP Tradicional & 7 & 5 & 12 \\
Chen et al (2009) & DP & 7 & 1 & 8 \\
Stockstrond e Herstatt (2008) & DP & 5 & 2 & 7 \\
Johnson et al (2009) & DP & 6 & 0 & 6 \\
Olausson e Berggren (2010) & DP & 1 & 0 & 1 \\
Hoda, Noble e Marshall (2010) & GP Ágil & 1 & 0 & 1 \\
Driessen e Ende (2010) & Organizações & 0 & 0 & 0 \\
Vinodh et al (2010) & Organizações & 0 & 0 & 0 \\
\hline Legenda: Google Scholar (GS); ISI Web of Knownledge (ISI); Desenvolvimento de Produtos (DP); \\
Gerenciamento de Projetos (GP) & & & &
\end{tabular}

\section{RESULTADOS}

O principal resultado deste estudo é uma lista que contém os FCAs que podem ser úteis em testes empíricos para melhor compreensão dos seus efeitos no desempenho das práticas, técnicas e ferramentas de gestão adotadas no DP, segundo uma abordagem ágil de GP. Os FCAs foram agrupados em 4 categorias: organização, processo, time de projeto e produto/projeto. Os resultados serão apresentados seguindo as categorias. Estratégias semelhantes de categorização de fatores foram adotadas em outros estudos, como Chen et al (2009) e Chow e Cao (2008).

\subsection{Organização}

A categoria organização foi a que obteve maior número de FCAs identificados (Tabela 2). No total foram 13 dos quais quatro se destacaram, seja pela extensão do conceito ou pelo número de autores que os citam.

Tabela 2 - Fatores críticos da agilidade classificados na categoria "organização".

\begin{tabular}{llllll}
\hline $\mathbf{n}^{\circ}$ & \multicolumn{1}{c}{ Fator } & \multicolumn{1}{c}{ Autores (Ano) } & \multicolumn{1}{c}{ Área } & GS & ISI \\
\hline \multirow{3}{*}{1} & Bustelo, Avella e Fernández & Manufatura & 13 & 9 \\
\cline { 3 - 6 } & Estrutura & $(2007)$ & DP & 7 & 1 \\
\cline { 2 - 6 } & Organizacional & Chen et al (2009) & GP Tradicional & 7 & 5 \\
\cline { 2 - 6 } & Liu e Yetton (2007) & Organizações & 0 & 0 \\
\cline { 2 - 6 } & & &
\end{tabular}




\begin{tabular}{|c|c|c|c|c|c|}
\hline \multirow{3}{*}{2} & & Chow e Cao (2008) & Software & 68 & 18 \\
\hline & \multirow{2}{*}{$\begin{array}{l}\text { Cultura } \\
\text { Organizacional }\end{array}$} & $\begin{array}{l}\text { Bustelo, Avella e Fernández } \\
\text { (2007) }\end{array}$ & Manufatura & 13 & 9 \\
\hline & & Chow e Cao (2008) & Software & 68 & 18 \\
\hline 3 & Empreendedorismo & $\begin{array}{l}\text { Bustelo, Avella e Fernández } \\
\text { (2007) }\end{array}$ & Manufatura & 13 & 9 \\
\hline & \multirow{4}{*}{$\begin{array}{l}\text { Aprendizado } \\
\text { Organizacional }\end{array}$} & $\begin{array}{l}\text { Bustelo, Avella e Fernández } \\
(2007)\end{array}$ & Manufatura & 13 & 9 \\
\hline 4 & & $\begin{array}{l}\text { Yusuf, Sarhadi e } \\
\text { Gunasekaran (1999) }\end{array}$ & $\begin{array}{l}\text { Cadeia de } \\
\text { Suprimentos }\end{array}$ & 263 & 111 \\
\hline & & $\begin{array}{l}\text { Lynn et al (2000) apud } \\
\text { Chen et al (2009) }\end{array}$ & $\mathrm{DP}$ & 92 & 45 \\
\hline & & Gehani (1995) & Organizações & 32 & 15 \\
\hline 5 & $\begin{array}{l}\text { Ambiente de } \\
\text { trabalho que } \\
\text { estimule métodos } \\
\text { ágeis }\end{array}$ & Chow e Cao (2008) & Software & 68 & 18 \\
\hline 6 & $\begin{array}{l}\text { Métodos ágeis bem } \\
\text { aceitos }\end{array}$ & Chow e Cao (2008) & Software & 68 & 18 \\
\hline 7 & $\begin{array}{l}\text { Recompensa } \\
\text { apropriada para } \\
\text { métodos ágeis }\end{array}$ & Chow e Cao (2008) & Software & 68 & 18 \\
\hline \multirow{3}{*}{8} & \multirow{3}{*}{$\begin{array}{l}\text { Enfase em } \\
\text { velocidade }\end{array}$} & Chow e Cao (2008) & Software & 68 & 18 \\
\hline & & $\begin{array}{l}\text { Kessler e Chakrabarti (1996) } \\
\text { apud Chen et al (2009) }\end{array}$ & $\mathrm{DP}$ & 348 & 155 \\
\hline & & Johnson et al (2009) & $\mathrm{DP}$ & 6 & 0 \\
\hline 9 & $\begin{array}{l}\text { Comprometimento } \\
\text { do time em tempo } \\
\text { integral }\end{array}$ & Chow e Cao (2008) & Software & 68 & 18 \\
\hline \multirow{6}{*}{10} & \multirow{6}{*}{$\begin{array}{l}\text { Times } \\
\text { multifuncionais }\end{array}$} & $\begin{array}{l}\text { Bustelo, Avella e Fernández } \\
\text { (2007) }\end{array}$ & Manufatura & 13 & 9 \\
\hline & & Gehani (1995) & Organizações & 32 & 15 \\
\hline & & $\begin{array}{l}\text { Yusuf; Sarhadi e } \\
\text { Gunasekaran (1999) }\end{array}$ & $\begin{array}{l}\text { Cadeia de } \\
\text { Suprimentos }\end{array}$ & 263 & 111 \\
\hline & & Verganti (1999) & DP & 89 & 26 \\
\hline & & Zirger e Hartley (1996) & $\mathrm{DP}$ & 173 & 84 \\
\hline & & Olausson e Berggren (2010) & $\mathrm{DP}$ & 1 & $\mathrm{NE}$ \\
\hline 11 & $\begin{array}{l}\text { Competição por } \\
\text { Recursos }\end{array}$ & Matson e McFarlane (1999) & Manufatura & 46 & 16 \\
\hline \multirow{4}{*}{12} & \multirow{4}{*}{$\begin{array}{l}\text { Apoio executivo } \\
\text { forte }\end{array}$} & Zirger e Hartley (1996) & $\mathrm{DP}$ & 173 & 84 \\
\hline & & Chow e Cao (2008) & Software & 68 & 18 \\
\hline & & $\begin{array}{l}\text { Bustelo, Avella e Fernández } \\
\text { (2007) }\end{array}$ & Manufatura & 13 & 9 \\
\hline & & Chen et al (2009) & $\mathrm{DP}$ & 7 & 1 \\
\hline 13 & $\begin{array}{l}\text { Tomada de decisão } \\
\text { descentralizada }\end{array}$ & Chen et al (2009) & DP & 7 & 1 \\
\hline
\end{tabular}

Legenda: Desenvolvimento de Produtos (DP); Google Scholar (GS); ISI Web of Knownledge (ISI); Não Encontrado (NE) 
O fator que teve maior extensão e número de citações foi "times multifuncionais". É aconselhável a formação de times por membros de diversas áreas da organização no desenvolvimento de projetos inovadores. A diversidade das competências e departamentos contribuiu para a interação e, desse modo, pode aumentar a comunicação e integração de objetivos entre os grupos funcionais pela redução de conflitos entre as áreas envolvidas no projeto. Isso pode ter um impacto positivo, por exemplo, na redução do ciclo de vida do projeto, conforme discutido por Zirger e Hartley (1996). O uso de times multifuncionais tem sido estudado há bastante tempo, principalmente em trabalhos relacionados ao desenvolvimento de produtos (BARCZAK; GRIFFIN; KAHN, 2009).

O FCA "estrutura organizacional" também teve grande destaque, tanto em relação ao número de citações quanto na extensão do conceito. Shenhar (2001) argumenta que uma organização "orgânica" - caracterizada por ser informal e descentralizada e com poucos níveis de autoridade - é melhor indicada para lidar com as incertezas inerentes de ambientes dinâmicos de negócio. Em contrapartida, as organizações "mecânicas" - centralizadas, especializadas e burocráticas, teriam melhor desempenho em ambientes previsíveis. O "aprendizado organizacional" é um conceito que se destacou principalmente com relação à extensão. Quanto maior o nível de aprendizado maior a eficiência e eficácia dos times para atender os objetivos de velocidade do DP (LYNN et al, 2000; SARIN; MCDERMOTT, 2003 apud CHEN et al, 2009). Por último, o fator "apoio executivo" (ou apoio da alta gerência) também se destacou. O apoio da alta gerência facilita a superação dos obstáculos e proporciona acesso aos recursos humanos e financeiros além de motivar os integrantes da equipe de projetos (SWINK, 2003).

Merecem destaque também os FCAs citados principalmente na área de software. Por exemplo, os fatores "ambiente de trabalho que estimule o uso de métodos ágeis", "métodos ágeis bem aceitos" e "recompensa pelo uso dos métodos ágeis", citados por Chow e Cao (2008), podem ser relevantes para o uso da abordagem ágil em outros ambientes, como no desenvolvimento de produtos manufaturados. A presença desses fatores deve ser melhor compreendida e investigada. Uma vez que a abordagem ágil propõe um conjunto de valores e preconiza o uso de práticas e ferramentas não triviais para as organizações, é preciso estimular o uso e a busca por conhecimento aprofundado sobre o GAP. Outro fator que se insere nesse contexto é a "ênfase em velocidade" citado por Chow e Cao (2008), Kessler e Chakrabarti apud Chen et al (2009) e Johnson et al (2009).

O fator "comprometimento do time" também demonstrou relevância para a área de software. Citado por Chow e Cao (2008), este fator pode ser confundido com a "dedicação do time", porém, nesse caso o primeiro está relacionado com o comprometimento com resultados e não o tempo total dedicado a um ou mais projetos em desenvolvimento. Nesse sentido, um time de projeto comprometido com os resultados pode contribuir positivamente para a adoção de determinadas práticas de gestão e consequentemente melhorar as possibilidades de sucesso nos projetos.

\subsection{Processo}

Nessa categoria, dez fatores foram selecionados, conforme Tabela 3. Desses, apenas dois foram mais expressivos em quantidade de citações e extensão, sendo 
eles: "fácil acesso a informações e banco de dados integrados" e a "execução de processos em paralelo". O fator "fácil acesso a informações e banco de dados integrados" pode contribuir para economizar tempo no armazenamento e recuperação de informações sobre o projeto, além de contribuir para a autonomia da equipe de projetos no uso dessas informações. Além disso, a integração dos bancos de dados contribui para a melhor gestão de configuração e melhoria da flexibilidade, uma vez que qualquer mudança é comunicada a todos os integrantes de imediato, facilitando sua compreensão e absorção.

O fator "execução de processos em paralelo" ou "simultaneidade" também tem impacto positivo em termos de uso de recursos no gerenciamento ágil de projetos. Isso porque realizar processos em paralelo pode contribuir para reduzir obstáculos entre funções e identificar e definir ações para problemas em potencial de forma antecipada (SWINK, 2003; ZIRGER; HARTLEY, 1996 apud CHEN et al, 2009). No estudo conduzido por Chen et al (2009), que investigou a relação entre diversos fatores e a velocidade do desenvolvimento de produtos, este fator apresentou correlação positiva.

Tabela 3 - Fatores críticos da agilidade classificados na categoria "processo"

\begin{tabular}{|c|c|c|c|c|c|}
\hline$n^{\circ}$ & Fator & Autores (Ano) & Área & GS & ISI \\
\hline 1 & $\begin{array}{l}\text { Capacidade de } \\
\text { reconfiguração }\end{array}$ & $\begin{array}{l}\text { Yusuf; Sarhadi e } \\
\text { Gunasekaran (1999) }\end{array}$ & $\begin{array}{l}\text { Cadeia de } \\
\text { Suprimentos }\end{array}$ & 263 & 111 \\
\hline 2 & $\begin{array}{l}\text { Automatização do } \\
\text { processo }\end{array}$ & Vinodh et al (2010) & Organizações & 0 & 0 \\
\hline 3 & $\begin{array}{l}\text { Modularidade do } \\
\text { processo }\end{array}$ & Eppinger e Chitkara (2009) & DP & 0 & 16 \\
\hline \multirow[t]{2}{*}{4} & \multirow{2}{*}{$\begin{array}{l}\text { Dados integrados e de } \\
\text { fácil acesso }\end{array}$} & $\begin{array}{l}\text { Bustelo, Avella e Fernández } \\
\text { (2007) }\end{array}$ & Manufatura & 13 & 9 \\
\hline & & Olausson e Berggren (2010) & DP & 1 & NE \\
\hline 5 & $\begin{array}{l}\text { Formalização do } \\
\text { processo }\end{array}$ & $\begin{array}{l}\text { Li e Atuahene-Gima (1996) } \\
\text { apud Chen et al (2009) }\end{array}$ & DP & 14 & NE \\
\hline \multirow[t]{2}{*}{6} & \multirow[t]{2}{*}{ Processos em paralelo } & $\begin{array}{l}\text { Tatikonda e Montoya-Weiss } \\
\text { (2001) apud Chen et al (2009) }\end{array}$ & DP & 180 & 89 \\
\hline & & Zirger e Hartley (1996) & DP & 173 & 84 \\
\hline 7 & Integração externa & $\begin{array}{l}\text { Yusuf; Sarhadi e } \\
\text { Gunasekaran (1999) }\end{array}$ & $\begin{array}{l}\text { Cadeia de } \\
\text { Suprimentos }\end{array}$ & 263 & 111 \\
\hline 8 & $\begin{array}{l}\text { Sistemas de suporte } \\
\text { (CAD; CAE; RP) }\end{array}$ & Verganti (1999) & DP & 89 & 26 \\
\hline \multirow{4}{*}{9} & \multirow{4}{*}{$\begin{array}{l}\text { Envolvimento do } \\
\text { cliente }\end{array}$} & Stockstrond e Herstatt (2008) & $\mathrm{DP}$ & 5 & 2 \\
\hline & & $\begin{array}{l}\text { Hoda, Noble e Marshall } \\
\text { (2010) }\end{array}$ & GP Ágil & 1 & 0 \\
\hline & & Chow e Cao (2008) & Software & 68 & 18 \\
\hline & & Chen et al (2009) & DP & 7 & 1 \\
\hline 10 & $\begin{array}{l}\text { Envolvimento dos } \\
\text { fornecedores }\end{array}$ & $\begin{array}{l}\text { Zirger e Hartley (1996) } \\
\text { Chen et al (2009) }\end{array}$ & $\begin{array}{l}\mathrm{DP} \\
\mathrm{DP}\end{array}$ & 173 & $\begin{array}{l}84 \\
1\end{array}$ \\
\hline
\end{tabular}

Legenda: Desenvolvimento de Produto (DP); Google Scholar (GS); ISI Web of Knowledge (ISI); Não Encontrado (NE)

Os resultados indicam a necessidade de melhor investigar dois fatores, são eles: "Modularidade do processo" (EPPINGER; CHITKARA, 2009) e "Formalização 
do processo" (LI; ATUAHENE-GINA, 1999 apud CHEN et al, 2009). Esses dois fatores em conjunto podem significar ganhos em velocidade no desenvolvimento do produto, redução de custos e menor uso de recursos para absorver mudanças durante o DP em ambientes caracterizados por mudanças constantes. A "formalização do processo" é considerada uma boa prática de desenvolvimento de produtos, conforme já evidenciado na teoria (BARCZAK; GRIFFIN; KAHN, 2009). Entretanto, merece ser investigado qual o nível de formalização adequado para ambientes dinâmicos de negócio que adotam a abordagem ágil de gerenciamento de projetos.

Entretanto, o nível de "formalização do processo" pode significar impactos positivos ou negativos no que tange a "modularidade do processo". Por exemplo, no caso de um projeto de desenvolvimento de produtos em nível global, no qual várias equipes trabalham de forma simultânea em diferentes partes do mundo, a formalização do processo é importante para garantir melhor interface e integração dos processos, ou "módulos de processo" (EPPINGER; CHITKARA, 2009). Já uma formalização excessiva, principalmente quando as equipes estão co-localizadas e possuem alto nível de interação, e autogestão, pode deixar o processo de DP demasiadamente burocrático, reduzindo a flexibilidade e a capacidade de resposta da equipe de projeto frente às mudanças do projeto.

Por fim, merece atenção um fator preponderante em projetos de desenvolvimento de produtos. Trata-se do envolvimento de fornecedores e/ou parceiros já nas fases iniciais do projeto, mas também durante seu desenvolvimento. Este fator foi citado por Zirger e Hartley (1996) e Chen et al (2009), mas tem sido discutido há um bom tempo por autores como Eisenhardt e Tabrizi (1995). No entanto, esse fator tem sido pouco explorado nos textos mais recentes sobre gerenciamento ágil de projetos (AUGUSTINE, 2005; CHIN, 2004; COHN, 2005; HIGHSMITH, 2004) devido seu enfoque na área de software.

\subsection{Time de Projeto}

Nessa categoria foram identificados oito fatores, apresentados na Tabela 4. Dentre esses, quatro se destacaram em extensão e número de citações, sendo eles: empowerment, dedicação, localização e experiência do time de projetos.

Tabela 4 - Fatores críticos da agilidade classificados na categoria "time de projeto"

\begin{tabular}{|c|c|c|c|c|c|}
\hline$n^{\circ}$ & Fator & Autores (Ano) & Área & GS & ISI \\
\hline 1 & Auto gestão & $\begin{array}{l}\text { Bustelo; Avella e Fernández } \\
\text { (2007) }\end{array}$ & Manufatura & 13 & 9 \\
\hline \multirow{6}{*}{2} & \multirow{6}{*}{ Empowerment } & $\begin{array}{l}\text { Bustelo; Avella e Fernández } \\
\text { (2007) }\end{array}$ & Manufatura & 13 & 9 \\
\hline & & Gehani (1995) & Organizações & 32 & 15 \\
\hline & & $\begin{array}{l}\text { Yusuf; Sarhadi e Gunasekaran } \\
\text { (1999) }\end{array}$ & $\begin{array}{l}\text { Cadeia de } \\
\text { Suprimentos }\end{array}$ & 263 & 111 \\
\hline & & Verganti (1999) & DP & 89 & 26 \\
\hline & & $\begin{array}{l}\text { Kessler e Chakrabarti (1996) } \\
\text { apud Chen et al (2009) }\end{array}$ & DP & 348 & 155 \\
\hline & & Zirger e Hartley (1996) & DP & 173 & 84 \\
\hline
\end{tabular}




\begin{tabular}{|c|c|c|c|c|c|}
\hline 3 & Liderança & $\begin{array}{l}\text { Sheremata (2000) apud Chen et } \\
\text { al (2009) }\end{array}$ & DP & 193 & 75 \\
\hline \multirow{5}{*}{4} & \multirow{5}{*}{ Dedicação } & $\begin{array}{l}\text { Bustelo; Avella e Fernández } \\
\text { (2007) }\end{array}$ & Manufatura & 13 & 9 \\
\hline & & Chow e Cao (2008) & Software & 68 & 18 \\
\hline & & Zirger e Hartley (1996) & DP & 173 & 84 \\
\hline & & Chen et al (2009) & DP & 7 & 1 \\
\hline & & Smith e Reinertsen (1992) & $\mathrm{DP}$ & 79 & 22 \\
\hline 5 & $\begin{array}{l}\text { Conhecimento } \\
\text { sobre métodos } \\
\text { ágeis }\end{array}$ & Chow e Cao (2008) & Software & 68 & 18 \\
\hline \multirow{3}{*}{6} & \multirow{3}{*}{$\begin{array}{l}\text { Experiência e } \\
\text { expertise }\end{array}$} & $\begin{array}{l}\text { Yusuf; Sarhadi e Gunasekaran } \\
\text { (1999) }\end{array}$ & $\begin{array}{l}\text { Cadeia de } \\
\text { Suprimentos }\end{array}$ & 263 & 111 \\
\hline & & Chow e Cao (2008) & Software & 68 & 18 \\
\hline & & Chen et al (2009) & DP & 7 & 1 \\
\hline \multirow{2}{*}{7} & \multirow{2}{*}{ Tamanho } & Smith e Reinertsen (1992) & $\mathrm{DP}$ & 79 & 22 \\
\hline & & Chow e Cao (2008) & Software & 68 & 18 \\
\hline \multirow{5}{*}{8} & \multirow{5}{*}{ Localização } & Verganti (1999) & DP & 89 & 26 \\
\hline & & Cornell e Dillon (2001) & GP Tradicional & 13 & 10 \\
\hline & & Zirger e Hartley (1996) & DP & 173 & 84 \\
\hline & & Chow e Cao (2008) & Software & 68 & 18 \\
\hline & & Chen et al (2009) & DP & 7 & 1 \\
\hline
\end{tabular}
Encontrado (NE)

O empowerment foi o fator que teve a maior extensão e número de citações. Esse fator é definido como o grau de autonomia do time de projeto (KESSLER; CHAKRABARTI, 1996). No estudo conduzido por Chen et al (2009) esse fator apresentou correlação positiva com a velocidade no desenvolvimento de produtos.

A "dedicação do time" também se destacou e é um fator preconizado na teoria de gerenciamento ágil de projetos (HIGHSMITH, 2004). Para o autor trabalhar com times dedicados é um fator preponderante para melhor aplicar as práticas do GAP. Times pequenos e dedicados à apenas um projeto por vez é visto como algo benéfico segundo a teoria GAP (HIGHSMITH, 2004). Na literatura esse fator é bastante explorado e existem formas de mensurá-lo, por exemplo: pela porcentagem de funcionários trabalhando em tempo integral no projeto ou pela baixa rotatividade de membros da equipe (CARBONELL; RODRIGUEZ, 2006; KELLER, 2008).

Outro fator relevante na utilização da abordagem de gerenciamento ágil é a "localização do time do projeto" (HIGHSMITH, 2004; MAFAKHERI; NASIRI; MOUSAVI, 2008; QUMER; SELLERS, 2008; ALMEIDA et al, 2010). A proximidade dos membros do time é considerada um fator que contribui positivamente para o aumento da agilidade do desenvolvimento e melhor comunicação entre o gerente de projetos e membros da equipe. Na teoria de GAP aconselha-se inclusive a colocalização, ou seja, os membros da equipe de projeto trabalhando em uma mesma sala, facilitando a condução de reuniões formais ou informais e interações frequentes para a discussão e definição de soluções para os problemas e mudanças no projeto (CARBONELL; RODRIGUEZ, 2006; ZIRGER; HARTLEY, 1996).

O estudo de Barczak, Griffin e Kahn (2009) evidenciou a utilização de "times co-localizados" e a realização de reuniões presenciais (face-to-face), como 
estratégias bastante comuns no desenvolvimento de produtos, segundo uma pesquisa realizada com 410 profissionais de indústrias de setores como bens de capital, químico, software e hardware, serviços e saúde. Entretanto é preciso considerar o uso de tecnologias de comunicação em tempo real, que podem ser utilizadas em times "virtuais", que não estejam próximos, facilitando assim a troca de informações e melhor comunicação (BARCZAK; GRIFFIN; KAHN, 2009). Muito embora sejam úteis, essas ferramentas não substituem integralmente a interação presencial entre os membros de um time de projetos, facilitada pela co-localização.

Por fim, o FCA "experiência e conhecimento do time", tanto com relação ao tipo de projeto como o método de gerenciamento escolhido, pode ser considerado um fator relevante, conforme evidenciado na teoria. A experiência do time possibilita a identificação antecipada de problemas e a definição de práticas e técnicas adequadas e compatíveis com os objetivos do projeto que sejam úteis na resolução dos dificuldades e conflitos no DP, contribuindo para a redução do ciclo de vida de desenvolvimento (SWINK, 2003; CARBONELL; RODRIGUEZ, 2006 apud CHEN et al, 2009).

\subsection{Produto/projeto}

A categoria "produto/projeto" teve cinco fatores identificados, mas apenas dois demonstraram maior relevância na amostra de artigos considerada neste estudo (Tabela 5) sendo que eles estão diretamente relacionados ao ambiente turbulento de negócios nos quais o GAP se diz mais eficiente. Por exemplo, o FCA "clareza dos objetivos" é considerado importante nesses ambientes nos quais as incertezas e riscos são altos. Este fator contribui para que o time de projetos mantenha o foco, evitando mudanças de direção e retrabalho (SWINK, 2003). Esse fator também foi identificado como sendo relevante no estudo de Barczak, Griffin e Kahn (2009).

Da mesma forma o "grau de inovação" do projeto também pode estar diretamente relacionado aos ambientes incertos, com a tecnologia adotada, sendo fonte das principais causas das incertezas no DP (TATIKONDA; ROSENTHAL, 2000). Quanto mais inovador o produto a ser desenvolvido, mais flexível deve ser o processo de gestão a fim de absorver as mudanças de escopo e colocar os planos de contingência em prática de maneira mais rápida e eficaz.

Tabela 5 - Fatores críticos da agilidade classificados na categoria "produto/projeto"

\begin{tabular}{|c|c|c|c|c|c|}
\hline$n^{\circ}$ & Fator & Autores (Ano) & Área & GS & ISI \\
\hline 1 & $\begin{array}{l}\text { Plano de sucessão dos } \\
\text { produtos }\end{array}$ & Gehani (1995) & Organizações & 32 & 15 \\
\hline 2 & Criticidade do projeto & Chow e Cao (2008) & Software & 68 & 18 \\
\hline \multirow{3}{*}{3} & \multirow{3}{*}{ Clareza dos objetivos } & Chen et al (2009) & DP & 7 & 1 \\
\hline & & Liu e Yetton (2007) & $\begin{array}{l}\text { GP } \\
\text { Tradicional }\end{array}$ & 7 & 5 \\
\hline & & $\begin{array}{l}\text { Stockstrond e Herstatt } \\
(2008)\end{array}$ & DP & 5 & 2 \\
\hline 4 & $\begin{array}{l}\text { Complexidade do } \\
\text { projeto }\end{array}$ & $\begin{array}{l}\text { Swink (2003) apud Chen et } \\
\text { al (2009) }\end{array}$ & DP & 37 & 13 \\
\hline \multirow{2}{*}{5} & \multirow{2}{*}{$\begin{array}{l}\text { Grau de inovação do } \\
\text { projeto }\end{array}$} & Chow e Cao (2008) & Software & 68 & 18 \\
\hline & & Chen et al (2009) & DP & 7 & 1 \\
\hline
\end{tabular}


Chen et al (2005)

$\mathrm{DP}$

$72 \quad 23$

Legenda: Desenvolvimento de Produto (DP); Google Scholar (GS); ISI Web of Knownledge (ISI); Não Encontrado (NE)

\section{CONSIDERAÇÕES FINAIS}

Esse estudo reúne 36 fatores críticos da agilidade que podem impactar direta ou indiretamente no desempenho do processo de gerenciamento ágil de projetos de desenvolvimento de novos produtos. Esse resultado foi obtido por meio de uma revisão bibliográfica sistemática que investigou artigos de áreas que também adotam o conceito de agilidade como: manufatura, organizações, cadeia de suprimentos, gerenciamento de projetos (ágil e tradicional), software e desenvolvimento de produtos. Observou-se que a categoria "organização" teve a maior contribuição em número de fatores críticos da agilidade, totalizando treze fatores identificados. As categorias "processo", "time de projeto" e "produtos/projetos", contribuíram com dez, oito e cinco fatores respectivamente.

Dos 36 fatores estudados, 12 foram considerados relevantes segundo os critérios adotados no estudo (quantidade de citações dos artigos no ISI e GS e número de áreas em que os fator aparece) sendo esses: times multifuncionais, estrutura organizacional, aprendizado organizacional, apoio executivo, fácil acesso a informação e banco de dados integrados, execução de processos em paralelo, empowerment, dedicação, localização, experiência do time de projetos, clareza nos objetivos e grau de inovação do produto/ software.

$\mathrm{Na}$ análise dos fatores, segundo o critério "extensão do conceito", destacou os fatores "estrutura organizacional", "times multifuncionais", "empowerment", "apoio executivo forte" e "dados integrados e de fácil acesso". Esse resultado reforça a necessidade de melhor investigá-los, pois há indícios de que esses fatores podem influenciar na adoção de práticas de gerenciamento ágil de projetos e consequentemente no desempenho dessas práticas.

Já os fatores "clareza nos objetivos", "grau de inovação", "localização da equipe", "experiência do time" e "dedicação" tiveram várias menções, inclusive de autores da área de desenvolvimento de produtos, apontando para uma forte influência dos mesmos. Os resultados da pesquisa conduzida por Barczak, Griffin e Kahn (2009) corroboram com as evidencias encontradas nesse estudo, enfatizando a necessidade de melhor investigar esses fatores e sua correlação com o desempenho do processo de gerenciamento de projetos baseado na adoção de práticas "ágeis" conforme preconizadas na teoria do GAP.

Como limitações para esse trabalho aponta-se que o mesmo ainda está em andamento e outros estudos estão sendo considerados como fonte para a identificação e análise dos fatores críticos da agilidade. Além disso, é importante ressaltar que essa lista precisa ser refinada. Existem fatores que podem ser confundidos com práticas de gestão. Há ainda fatores que podem ser tanto uma prática quanto um fator. Citam-se como exemplos os fatores 3 e 6 (Tabela 3), "modularidade do processo" e "processos em paralelo", respectivamente. Ambos os fatores podem ser confundidos como uma prática de gestão. Entretanto, é preciso compreender se essas práticas podem ser aplicadas pela organização, ou seja, está sob seu controle e não depende de nenhum outro fator interno ou externo. 
Outros fatores que podem ser confundidos com práticas de gestão são "envolvimento do cliente" e "envolvimento de fornecedores" (Tabela 3). Nesses casos, é importante analisar se o tipo de projeto e o segmento de atuação da empresa possibilitam envolver o cliente e fornecedores. Há casos em que o envolvimento desses autores não é possível. Em outros casos, as empresas tem possibilidade de envolver os atores, entretanto, deixam de fazer pela ausência de práticas ou processo. Em ambas as situações o "envolvimento do cliente" e "envolvimento de fornecedores" podem ser considerados fatores críticos da agilidade.

Nesse estudo considerou-se trabalhos de diversas áreas como manufatura, organizações e cadeia de suprimentos, que utilizaram o conceito de agilidade como base para sua investigação. Há, portanto, a necessidade de ampliar a quantidade de estudos, principalmente considerando outras fontes como livros e artigos recentes publicados em anais de congresso. Por se tratar de um resultado parcial, este estudo focou apenas artigos completos publicados em periódicos científicos.

Como estudo futuro indica-se $o$ detalhamento e análise dos FCAs apresentados nessa pesquisa, bem como a comprovação empírica da existência desses fatores em casos reais de desenvolvimento de produtos. O próximo passo desse estudo compreende a definição de formas de medição desses fatores e a observação em casos reais que adotam práticas segundo a abordagem de gerenciamento ágil de projetos.

\section{AGRADECIMENTOS}

Os autores agradecem o apoio financeiro à pesquisa oferecido pela FAPESP e CAPES, sem o qual não seria possível a realização deste trabalho.

\section{REFERÊNCIAS}

ALMEIDA, L. F. M.; CONFORTO, E. C.; FARINELLO, E. L.; REGANATI, M. R. P. L.; SILVA, M. A. Nível de agilidade no planejamento de projetos: comparação em uma empresa de tecnologia da informação. XVII Simpósio Brasileiro de Engenharia de Produção, Novembro de 2010, UNESP, Bauru, p. 1-15, 2010.

AMARAL, D. C.; CONFORTO, E. C.; BENASSI, J. L. G.; ARAÚJO, C. Gerenciamento ágil de projetos - aplicação em produtos inovadores. São Paulo: Editora Saraiva, 2011.

AUGUSTINE, S. Managing Agile Projects. Virginia: Prentice Hall PTR, 2005.

BARCZAK, G.; GRIFFIN, A.; KAHN, K. B. Perspective: trends and drives of success in NPD pratices: results of the 2003 PDMA best pratices study. Journal of Product Innovation Management, v. 26, p. 3-23, 2009. 
BECK, K.; BEEDLE, M.; VAN BENNEKUM, A.; et al. Manifesto for agile software development. 2001. Disponível em http://www.xprogramming.com/publications/dc9810cs.pdf Acesso em 16 jan. 2007.

BOTTTANI, E. A fuzzy QFD approach to achieve agility. International Journal of Production Economics, n. 119, p. 380-391, 2009.

BUSTELO, D. V.; AVELLA, L.; FERNÁNDEZ, E. Agility drives, enablers and outcomes: Empirical test of an integrated agile manufacturing model. International Journal of Operations \& Production Management, v. 119, n. 2, p. 380-391, 2009.

CARBONELL, P., RODRIGUEZ, A. I. Designing teams for speedy product development: themoderating effect of technological complexity. Journal of Business Research, 2006.

CHEN, J.; DAMANPOUR, F.; REILLY, R. R. Understanding antecedents of new product development speed: A meta-analysis. Journal of Operations Management, article in press, 2009.

CHEN, J.; REILLY, R. R.; LYNN, G. S. The impacts of speed-to-market on new product success: the moderating effects on uncertainty. IEEE Transactions on engineering management, v. 52, n. 2, 2005.

CHIN, G. Agile Project Management: how to succeed in the face of changing project requirements. New York: Amacom, 2004.

CHOW, T.; CAO, D. B. A survey of critical success factors in agile software projects. The Journal of Systems and Software, v. 81, p. 961-971, 2008.

COHN, M. Agile Estimating and Planning. New York: Prentice Hall PTR, 2005.

CONFORTO, E. C., AMARAL, D. C. Applying Agile Project Management approach to NPD: the case of a small technology-based enterprise. In: Proceeding of $16^{\text {th }}$ International Product Development Management Conference, 2009, Enschede/Netherlands, EIASM, 2009.

CONFORTO, E. C.; AMARAL, D. C. Evaluating an agile method for planning and controlling innovative projects. Project Management Journal, v. 41, n. 2, p. 73-80, 2010.

CONFORTO, E. C.; AMARAL, D. C. Agile Project Management and Innovative Product Development: benefits and challenges of two companies from São Carlos Tech-Pole, Brazil. In: 21st Annual Conference of the Production and Operations Management Society. Vancouver / British Columbia D: Production \& Operations Management Society - POMS, 2010.

CONFORTO, E. C.; AMARAL, D. C.; SILVA, S. L. Roteiro Para Revisão Bibliográfica Sistemática: Aplicação No Desenvolvimento De Produtos E Gerenciamento De Projetos. In: $8^{\circ}$. Congresso Brasileiro de Gestão de Desenvolvimento de Produto - 
CBGDP 2011, Porto Alegre, RS, Brasil. Instituto de Gestão de Desenvolvimento do Produto - IGDP. Anais... Porto Alegre: IGDP, 2011.

CORNELL, M. E. P.; DILLON, R. L. Success factors and future challenges in the management of faster-better-cheaper projects: lessons learned from NASA. IEEE Transactions Engineering Management, v. 48, n. 1, 2001.

DAWSON, R.; DAWSON, C. Practical proposals for managing uncertainty and risk in project planning. International Journal of Project Management, v. 16, n. 5, p. 299310, 1998.

DOVE, R. Knowledge management, response ability, and the agile enterprise. Journal of Knowledge Management, v. 3, n. 1; p. 18, 1999.

DRIESSEN, F. B.; ENDE, J. Innovation Management Pratices Compared: The Example of Project Based Firms. Journal of Product Innovation Management, v. 27, p. 705-724, 2010.

EBERT, C.; MAN, C. Effectively utilizing Project, product and process knowledge. Information and Software Technology, v. 50, p. 579-594, 2008.

EDER, S.; CONFORTO, E. C.; OLIVEIRA, M. G.; AMARAL, D. C.; SILVA, S. L., Estudo exploratório do conceito agilidade: modelo teórico para aplicação no gerenciamento ágil de projetos. XVII Simpósio Brasileiro de Engenharia de Produção, UNESP, Bauru, p. 1-15, nov. 2010.

EPPINGER, S. D.; CHITKARA, A. R. The practice of global product development. MITSloan Management Review, 2009.

EISENHARDT, K.; TABRIZI, B. N. Accelerating Adaptive Process: product innovation in the global computer industry. Administrative Science Quarterly, v. 40, n. 1, p. 84, 1995.

GANGULY, A.; NILCHIANI, R.; FARR, J. V. Evaluating agility in corporate enterprises. International Journal of Production Economics, v. 118, n. 2, p. 410-423, 2009.

GEHANI, R. R. Time-based management of technology - a taxonomic integration of tactical and strategic roles. International Journal of Operations \& Production Management, v. 15, n. 2, p. 19-35, 1995.

HIGHSMITH, J. Agile Project Management: creating innovative products. Boston: Addison-Wesley, 2004.

HODA, R.; NOBLE, J.; MARSHALL, S. The impact of inadequate customer collaboration on self organizing Agile Teams. Information and Software technology. v. 53, p. 521-534, 2001.

JOHNSON, W. H. A.; PICCOLOTTO, Z.; FILIPPINI, R. The impacts of time performance and market knowledge competence on new product success: an 
international study. IEEE Transactions on Engineering Management, v. 56, n. 2, 2009.

KELLER, R. T. Transformational leadership, initiating structure, and substitutes for leadership: a longitudinal study of research and development project team performance. Journal of Applied Psychology, v. 91, n. 1, p. 202-210, 2006.

KESSLER, E. H.; CHAKRABARTI, A. K. Innovation speed: a conceptual model of context, antecedents and outcomes. Academy Management Journal, v. 21, n. 4, p. 1143-1191, 1996.

KIOPPENBORG, T.; OPFER, W. The current state of project management research: trends, interpretations and predictions. Project Management Journal, v. 33, n. 2, p. 518, 2002.

KOLLTVEIT, B.; KARLSEN, J.; GRONHAUG, K. Perspectives on project management. International Journal of Project Management, v. 25, n. 1, p. 3-9, 2007.

LI, H., ATUAHENE-GIMA, K. Marketing's influence and new product performance in Chinese firms. Journal of International Marketing, v. 7, n. 1, p. 34-56, 1999.

LIU, L.; YETTON, P. The contingent effects on project performance of conducting project reviews and deploying project management offices. IEEE Transactions on engineering management, v. 54, n. 4, 2007.

LYNN, G. S., REILLY, R. R., AKGUN, A. E. Knowledge management in new product teams: practice and outcomes. IEEE Transactions on Engineering Management, $\mathrm{v}$. 47, n. 2, p. 221-231, 2000.

MAFAKHERI, F.; NASIRI, F.; MOUSAVI, M. Project Agility assessment: an integrated decision analysis approach. Production \& Planning Control, v. 19, n. 6, p. 567-576, 2008.

MATSON, J. B.; McFARLANE, D. C. Assessing the responsiveness of existing production operations. International Journal of Production and Operation Management, v. 19, n. 8, p. 765-784, 1999.

OLAUSSON, D.; BERGGREN, C. Managing uncertain, complex product development in high-tech firms: in search of controlled flexibility. R\&D Management, v. 40 , n. 4 , p. 383-399, 2010.

PERMINOVA, O.; GUSTAFSSON, M.; WIKSTRÖM, K. Defining uncertainty in projects - a new perspective. International Journal of Project Management, v. 26, n. 1, p. 73-79, 2008.

QUMER, A., HENDERSON-SELLERS, B. An evaluation of the degree of agility in six agile methods and its applicability for method engineering. Information and Software Technology, v. 50, p. 280-295, 2008. 
ROCKHART, J. F., CRESCENZI, A. D. Engaging top management in information technology. Sloan Management Review, v. 25, p. 3-16, 1984.

SARIN, S.; MCDERMOTT, C., The effect of team leader characteristics on learning, knowledge application ande performance of cross-functional nem product development teams. Decision sciences, v. 34, n. 4, p. 707-739, 2003.

SAUSER, B. J.; REILLY, R. R.; SHENHAR, A. J. Why projects fail? How contingency theory can provide new insights - A comparative analysis of NASA's Mars Climate Orbiter loss. International Journal of Project Management, v. 27, p. 665-679, 2009.

SHENHAR, A. J.; One size does not fit all projects: exploring classical contingency domains. Management Science, v. 47, n. 3, p. 394-414, 2001.

SHENHAR, A. J.; DVIR, D. Reinventing Project Management: the diamond approach to successful growth and innovation. Boston: Harvard Business School Press, 2007.

SHEREMATA, W. A. Centrifugal and centripetal forces in radical new product development under time pressure. Academy of Management Journal, v. 25, n. 2, p. 389-418, 2000.

SUIKKI, R.; THOMSTEDT, R.; HAAPASALO, H. Project management competence development framework in turbulent business environment. Technovation, v. 26, n. 5, p. 723-738, 2006.

SMITH, P. G.; REINERTSEN, D. G. Shortening the product development cycle. Research Technology Management, 1992.

STOCKSTROM, C.; HERSTATT, C. Planning and uncertainty in new product development. R\&D Management. v. 35, n. 8, p. 480-490, 2008.

SWINK, M. Completing projects on-time: how project acceleration affects new product development. Journal of Engineering and Technology Management, v. 20, p. 319-344, 2003.

TATIKONDA, M. V., MONTOYA-WEISS, M. M. Integrating operations and marketing perspectives of product innovation: the influence of organizational process factors and capabilities on development performance. Management Science, v. 47, n. 1, p. 151-172, 2001.

VERGANTI, R. Planned flexibility: linking anticipation and reaction in product development projects. Journal of Product Innovation Management, v. 16, p. 363-376, 1999.

VINODH, S.; DEVADASAN, S. R.; REDDY, B. V.; RAVICHAND, K. Agility index measurement using multi-grade fuzzy approach integrated in a 20 criteria agile model. International Journal of Production Research. v. 48, n. 23, p. 7159-7176, 2010. 
WILLIAMS, T. The need for new paradigms for complex projects. International Journal of Project Management, v. 17, n. 5, p. 269-273, 1999.

YUSUF, Y. Y.; SARHADI, M.; GUNASEKARAN, A. Agile Manufacturing: The drives, concepts and attributes. International Journal of Production Economics, v. 62, n. 1-2, p. 33-43, 1999.

ZHANG, Z. E.; SHARIFI, H. A methodology for achieving agility in manufacturing organizations. International Journal of Operations \& Production Management, v. 20, n. 4, p. 496-512, 1999.

ZIRGER, B. J.; HARTLEY, J. The effect of acceleration techniques on product development time, IEEE Transactions on engineering management, v. 43, n. 2, 1996. 